ELUA

ISSN 2171-6692

Núm. 36, 2021, págs. 89-107

https://doi.org/10.14198/ELUA.2021.36.05

\title{
Locuciones nominales complejas
}

\author{
Complex nominal idioms
}

\author{
RoQue LloRens García \\ Universidad Complutense de Madrid, España \\ rllorens@ucm.es \\ https://orcid.org/0000-0003-3500-3482
}

\section{Resumen}

El escaso consenso bibliográfico que existe sobre lo que es una locución, una colocación y un compuesto sintagmático plantea la necesidad de revisar el funcionamiento de cada uno de estos tipos de construcciones para tratar de identificar las características más relevantes que permitan discriminar un tipo de construcción de otro. En el presente trabajo presentamos una revisión sobre algunas características de las locuciones y las colocaciones que servirá para mostrar lo que entendemos por locución, y muy particularmente por locución nominal. A este respecto revisaremos una investigación de Barrios Rodríguez en la que pretende distinguir, mediante la prueba de la definición ostensiva, los compuestos sintagmáticos de las locuciones nominales. Por otra parte, también propone la clasificación de las locuciones nominales predicativas, distinguiéndolas de las locuciones nominales designativas (ampliamente estudiadas). Con el fin de valorar la viabilidad de estas propuestas de sistematización, hemos confeccionado un corpus en el que clasificamos compuestos sintagmáticos, locuciones nominales designativas y locuciones nominales predicativas. Al hilo del aná-

\begin{abstract}
The limited bibliographic consensus which exists regarding what an idiom, a collocation and a compound noun are, raises the requirement of reviewing the characteristics of each of these types of constructions in order to attempt to identify the most relevant features that allow distinguishing one type of construction from another. In this paper, a review of a number of characteristics of the collocations and idioms is presented. This review will assist in demonstrating what is understood by idiom, since idioms are the main type of construction that will be analysed. An investigation by Barrios Rodríguez in which she endeavours to distinguish, by means of the ostensive definition test, compound nouns from nominal idioms, will also be reviewed. Furthermore, the aforementioned investigation also proposes the classification of nominal idioms into two types (designative and predicative) according to how they behave as nouns or adjectives. In order to assess the viability of these systematization proposals, a commented classification of the different types of nominal constructions has been carried out. In addition, a new type of nominal idioms, with
\end{abstract}

Para citar este artículo: Llorens García, R. (2021). Locuciones nominales complejas. ELUA, (36), 89-107. https://doi.org/10.14198/ELUA.2021.36.05

Recibido: 31/07/2021, Aceptado: 07/10/2021

(C) 2021 Roque Llorens García

Este trabajo está sujeto a una licencia de Reconocimiento 4.0 Internacional de

Creative Commons (CC BY 4.0) 
lisis de los datos de nuestro corpus, proponemos un nuevo tipo de locuciones nominales cuyas propiedades y características se alejan tanto de las designativas como de las predicativas a las que hemos denominado locuciones nominales complejas. Los datos de nuestro corpus corroboran que la prueba de la definición ostensiva permite distinguir con precisión los compuestos sintagmáticos de las locuciones y colocaciones nominales. Asimismo, hemos comprobado que las locuciones nominales predicativas se diferencian sustancialmente de las designativas porque funcionan como adjetivos, de hecho, este tipo de locuciones pueden ser sustituidas en todos los casos por un adjetivo o por la correspondiente paráfrasis semántica de la locución. Las locuciones nominales complejas tienen una especial importancia en esta investigación porque ni siquiera están reconocidas como locuciones, $\mathrm{y}$ algunos autores afirman que su estudio corresponde a la sintaxis y no a la fraseología. A pesar de ello, consideramos que deben categorizarse como locuciones nominales y diferenciarse de los otros tipos de locuciones nominales.

PALABRAS CLAVE: compuesto sintagmático; locución nominal designativa; locución nominal predicativa; locución nominal compleja; definición ostensiva. characteristics that are very different from both the predicative and designative, is presented as what we have labelled complex nominal idioms. Finally, the paper arrives at the conclusion that the ostensive definition test allows the distinction of compound nouns from nominal collocations and idioms. Likewise, it has been verified that predicative nominal idioms differ substantially from designative ones due to the fact that they function as adjectives; in fact, these types of idioms can be replaced in all cases by an adjective or by the corresponding semantic paraphrase of the idiom. Complex nominal idioms have a significant importance in this investigation due to the fact that they are, in fact, not even recognized as idioms. Some authors affirm that these constructions belong to syntax and not to phraseology. Despite this, the fact that they should be studied as nominal idioms and should be differentiated from the other types is considered.

KEY WORDS: compound noun; designative nominal idiom; predicative nominal idiom; complex nominal idiom; ostensive definition.

\section{INTRODUCCIÓN}

En la presente investigación vamos a realizar diversas consideraciones sobre la compleja y debatida cuestión en torno a la distinción entre colocaciones nominales, locuciones nominales y compuestos sintagmáticos. La importancia de este tema estriba en el escaso consenso teórico que existe entre los distintos investigadores que abordan el tema. Frecuentemente, las mismas construcciones son clasificadas como colocaciones, locuciones o compuestos sintagmáticos dependiendo del marco teórico escogido por cada autor.

Es numerosa la bibliografía que recoge y repasa las distintas clasificaciones de las locuciones, aunque lo que predomina en estos estudios son las discrepancias ( $c f r$. Casares 1969; Mel'čuk 1996; Ruiz Gurillo 2001; García-Page 2008; Buenafuentes de la Mata 2010)ํ․ El marco teórico predominante que seguiremos es el propuesto por Barrios Rodríguez (2022) en el que propone la prueba de la definición ostensiva para distinguir las locuciones nominales de los compuestos sintagmáticos. Además plantea una reestructuración en la clasificación

1 No es el objetivo de esta investigación, en ningún caso, hacer un estudio bibliográfico sistemático de las distintas posturas y numerosísimas clasificaciones que han sido propuestas, sino destacar algunos ejemplos para mostrar, de forma práctica, el escaso acuerdo entre los diferentes estudios sobre los conceptos locución, colocación y compuesto sintagmático. 
de las locuciones nominales, en la que distingue, mediante una serie de pruebas semánticas y sintácticas, las locuciones nominales designativas de las que denomina locuciones nominales predicativas, que funcionan como adjetivos.

Para implementar su propuesta hemos creado un corpus en el que hemos aplicado las mencionadas pruebas. El corpus se ha confeccionado con los diccionarios de la Real Academia Española (2001) y de Seco et alii (2004). Además, hemos tomado ejemplos de Casares (1969), Bustos (1986), Buenafuentes de la Mata (2010), García-Page (2011) y Orduña (2019). Buena parte de los ejemplos los hemos recategorizado.

Este artículo se organiza del siguiente modo. En $\S 2$ repasamos los conceptos de colocación, locución y compuesto sintagmático en la bibliografía precedente y señalamos los principales problemas que impiden una distinción clara entre las locuciones y las colocaciones.

En $\S 3$ nos centramos en la compleja cuestión que supone la distinción entre locuciones nominales y compuestos sintagmáticos. Presentamos los datos de nuestro corpus de construcciones nominales, revisamos la propuesta de Barrios Rodríguez para distinguir los compuestos sintagmáticos de las locuciones nominales y planteamos la reestructuración de las locuciones nominales. En $\S 4$ presentamos nuestro análisis del corpus de construcciones nominales que hemos creado ad hoc con intención de verificar la utilidad y eficacia de la propuesta de Barrios Rodríguez. Por último, en $\S 4.4$ y $\S 5$, proponemos la existencia de otro tipo de locuciones, que hemos denominado locuciones nominales complejas, ya que no se ajustan a los criterios de clasificación de las que hemos revisado en los apartados precedentes.

\section{LOS CONCEPTOS DE LOCUCIÓN, COLOCACIÓN Y COMPUESTO SINTAG- MÁTICO}

\subsection{Estado de la cuestión}

La distinción entre los conceptos de locución, colocación y compuesto sintagmático es un problema que la bibliografía fraseológica no ha logrado resolver a pesar de los abundantes y extensos trabajos que existen sobre la cuestión. Trataremos de sintetizar a continuación algunos rasgos sobre los que se da un mínimo de acuerdo:

I. Locución: grupo de palabras lexicalizadas que constituyen una única pieza léxica y ejercen la misma función sintáctica que la categoría que les da nombre (RAE y Asociación de Academias de la Lengua Española 2009: 53). Ejemplos: para parar un tren, 'en abundancia'; costar un ojo de la cara, 'ser caro'.

II. Colocación: combinación de palabras no libre que puede estar asociada o no a un sentido figurado. Son construcciones que generalmente tienen un grado de fijación menor que las locuciones. Su restricción es léxica, permite la sustitución de algunos de sus elementos (Bosque 2004). Ejemplos: barra de pan, copo de agua, buen carácter, mal carácter.

III. Compuesto sintagmático: grupo de palabras yuxtapuestas que conservan su independencia gráfica y acentual (RAE y ASALE 2009: 735-736). Este tipo de cons-

2 Las paráfrasis semánticas que suceden a algunas construcciones, marcada al uso, con comillas simples, son originales nuestras si no se indica lo contrario. 
trucciones constituyen una unidad de denominación, es decir, aluden a una realidad concreta (Buenafuentes de la Mata 2010). Ejemplos: tocino de cielo, oro negro.

Teniendo en cuenta estas definiciones, es posible que se den ciertos casos de solapamiento entre las categorías de locuciones y colocaciones. Por ello, es conveniente señalar las características principales que comparten este tipo de construcciones: la combinación de dos o más palabras, la fijación y la idiomaticidad (Koike 2001) ${ }^{3}$. La complejidad de clasificar las distintas construcciones de forma sistemática estriba en que las características de estas construcciones no son completamente estables e identificables. El nivel de fijación de las construcciones puede ser gradual: lo divino y lo humano ${ }^{4}$ es una locución absolutamente fija y no admite la más mínima modificación; negocio redondo admite la flexión de número, pero a pesar de ello tiene un alto grado de fijación; metidos en un lío y metidos en un berenjenal son colocaciones porque permiten sustituir un sustantivo por otro, siendo, por tanto, unas construcciones con menor fijación que las anteriores.

En este estudio entendemos por composicionalidad la interpretación del significado de una construcción a través de la suma de significados de cada uno de los componentes de la construcción. Cuando interpretamos de manera literal una construcción o una parte de una construcción también podemos decir que es transparente. Por su parte, la idiomaticidad es lo opuesto a la composicionalidad. Una construcción idiomática no permite la interpretación de su significado mediante la suma de los significados de los componentes de la construcción. Media naranja es una locución idiomática, ya que a través de los significados de media y de naranja no podemos obtener el significado completo de la construcción que es 'pareja perfecta' (RAE y ASALE 2009: 736). Teniendo esto en cuenta, podríamos decir que idiomaticidad es sinónimo de sentido figurado. Sin embargo, García-Page (2008) niega que estos dos conceptos sean sinónimos.

Como veremos a continuación, el problema que plantea la distinción entre compuestos sintagmáticos y locuciones nominales es similar al que plantean las locuciones y las colocaciones, puesto que los compuestos sintagmáticos también son combinaciones de palabras fijas que pueden ser combinaciones idiomáticas o no.

\subsection{Los límites entre las locuciones y las colocaciones}

En primer lugar, y con respecto al concepto de composicionalidad con el que trabajamos (§ 2.1), debemos señalar que no coincidimos con el significado de composicionalidad que ofrece Mel'čuk (1996). En efecto, el autor defiende que las locuciones no son composicionales en ningún caso, y las clasifica en tres categorías según su grado de transparencia: «Three types of idioms can be distinguished in such a way: full idioms, semi-idioms and quasi-idioms. All of them are non-compositional, but the degree of their transparency varies» (Mel'čuk 1996: 37). Conviene que nos detengamos en la definición de los quasi-idioms que, según el autor, son las locuciones más débiles de todas, pues contienen el significado

3 La combinación de dos o más palabras, la fijación y la idiomaticidad solamente son algunas de las principales características sobre las que vamos a hablar. No obstante, cabe señalar que las locuciones y las colocaciones se asocian a otras características.

4 No hemos hallado ningún resultado en plural de esta construcción salvo el título de una obra de José María Vargas Vila (Los divinos y los humanos). 
de sus dos componentes léxicos, pero además, también crean un tercer significado: empezar una familia contiene los significados 'empezar' y 'familia', sin embargo, el conjunto de la construcción tiene el significado de 'concebir al primer hijo con un cónyuge'. Por su parte, García-Page (2008: 26-27) añade que «los fraseólogos se vienen decantando por el carácter opcional de dicha característica [idiomaticidad] dada la ingente cantidad de locuciones que carecen de significado traslaticio». Esto contrasta con la postura de Mel'čuk (1996), que considera que, si un frasema es composicional, es automáticamente una colocación.

También suscita todo tipo de planteamientos la fijación o estabilidad de las locuciones. Así, para Barrios Rodríguez (2015: 14-15), las locuciones tienen una estructura muy rígida, en oposición a las colocaciones que «se caracterizan por su mayor flexibilidad sintáctica». Esta mayor flexibilidad, como señala la autora, se refleja, por ejemplo, en la flexibilidad léxica, pues la colocación admite la sustitución de palabras: violar una norma, trasgredir una norma, romper una norma.

Por su parte, García-Page (2008) señala el rasgo de la fijación en las locuciones de forma menos estricta, incluso en algunas ocasiones pudiera parecer que el grado de libertad que pueden tener las locuciones supera al grado de fijación, como veremos a continuación en algunos ejemplos del autor.

En efecto, el autor señala que la fijación de las locuciones sí que admite la adición de palabras cuando tienen una función intensificadora. García-Page presenta, entre otros, estos dos ejemplos para mostrar la adición de términos con función intensificadora en las locuciones: tener (muy) mala uva y estar hasta los (mismísimos) cojones. Sin embargo, a nuestro juicio, estos dos ejemplos parecen contradictorios o, al menos, de razonamiento superficial y débil, pues no solo admiten la introducción de otro elemento en la construcción, sino que además ambas construcciones contienen elementos intercambiables: tener (muy) mala uva, tener (muy) mala leche, tener (muy) mala hostia, tener (muy) mal humor; estar hasta los (mismísimos) cojones, estar hasta la coronilla, estar hasta los huevos, estar hasta las narices, etc. Además, esta última construcción admite incluso la supresión de uno de sus componentes léxicos: estar hasta los mismísimos. Resulta evidente el contraste entre la supuesta fijación de las construcciones analizadas en comparación con otras construcciones como coser y cantar, corriente y moliente, de armas tomar, al pie de la letra, pez gordo, el qué dirán, manga ancha, farolillo rojo, el séptimo cielo, cacao mental, etc., que son locuciones con una estructura absolutamente fija y que no tienen toda una serie de variantes léxicas como las que hemos mencionado aquí.

La RAE y la ASALE se pronuncian sobre la polémica de las variantes en la Nueva gramática de la lengua española afirmando que las locuciones no admiten la sustitución de ninguna de sus palabras:

La productividad mínima la presentan las locuciones nominales, que rechazan incluso la sustitución de alguno de sus componentes por un sinónimo. Es locución, por ejemplo, cabo suelto ('circunstancia pendiente imprevista') pero constituye un grupo nominal, construido libremente con una pauta sintáctica, cabo desatado. Esta propiedad diferencia marcadamente las locuciones de las llamadas COLOCACIONES (en el sentido de 'combinaciones léxicas restringidas frecuentes y preferentes'), como en labor improba $\sim$ tarea improba trabajo improbo (RAE y ASALE 2009: 745).

Teniendo en cuenta todo lo expuesto, la postura de la RAE y la ASALE se aleja de las afirmaciones de García-Page en las que otorga a las locuciones la capacidad de tener una fi- 
jación flexible, llegando a admitir la sustitución de términos dentro de una locución. La RAE y la ASALE (2009) no solo se pronuncian sobre la incapacidad de las locuciones de poder sustituir sus componentes, sino que además afirman que las alteraciones gramaticales solo son admitidas por las colocaciones. Como resultado de este análisis, resultaría procedente clasificar como colocaciones los mencionados ejemplos que García-Page clasifica como locuciones (estar hasta los (mismísimos) cojones, tener (muy) mala uva).

Tras el repaso de las distintas posturas sobre los límites de las locuciones y las colocaciones, pensamos que se pone suficientemente de manifiesto que no se establece con claridad lo que distingue una locución de una colocación. Hemos observado que las principales características que comparten, como la idiomaticidad o la fijación, sí están reconocidas por los distintos autores, pero cada estudio interpreta y establece unos límites a cada una de estas características. Es por esto por lo que, para autores como Corpas (1996) o García-Page (2008), las colocaciones son sintagmas libres y composicionales. Sobre esta cuestión, García-Page (2008) sostiene una postura tan contundente que incluso afirma que las colocaciones son productos de la sintaxis y no deben estudiarse como unidades de la fraseología. Absolutamente contraria es la posición de Mel'čuk (2001), la RAE y la ASALE (2009) o Barrios Rodríguez (2015) que, como hemos mencionado en este apartado, tienen una concepción de la locución como de una construcción absolutamente fija mientras que la colocación la ven como una construcción con menor grado de fijación.

\section{LOCUCIONES NOMINALES Y COMPUESTOS SINTAGMÁTICOS}

\subsection{Los límites entre la locución nominal y el compuesto sintagmático}

$\mathrm{Si}$, como hemos visto, la distinción entre locuciones y colocaciones es una ardua tarea, en el caso de las construcciones nominales la dificultad aumenta, pues también es necesario distinguir locuciones y colocaciones de los compuestos sintagmáticos. Como veremos a continuación, no hay ningún consenso entre los estudiosos para la clasificación de todas estas últimas construcciones nominales. Como ilustra Barrios Rodríguez (2022), la polémica sobre estos límites se halla en ejemplos como perrito caliente, clasificado como palabra compuesta por García-Page, y tocino de cielo, clasificado por Casares como locución nominal denominativa. Estas dos construcciones son objetos comestibles del mundo real y son idiomáticas, por tanto, resulta contradictorio que se clasifique cada una de ellas de manera distinta.

Buenafuentes de la Mata (2010) señala que la fijación y la idiomaticidad son las dos características principales que comparten las locuciones y los compuestos sintagmáticos. También señala que son estas mismas características las que permiten diferenciar las locuciones de los compuestos sintagmáticos teniendo en cuenta la gradación, aunque admite que esta no es una prueba definitiva. Además, la autora asegura que una gran parte de los fraseólogos consideran que las locuciones tienen un mayor grado de idiomaticidad y fijación que los compuestos sintagmáticos. La utilidad de estas afirmaciones para distinguir los tipos de construcciones nominales, bajo nuestro punto de vista, es sin embargo cuestionable, prueba de ello son los numerosísimos casos que tradicionalmente se clasifican como compuestos sintagmáticos que son idiomáticos, incluso por la propia Buenafuentes de la Mata (2010): hombre rana, brazo de gitano, casco de burro, etc. Por lo general, la bibliografía (GarcíaPage 2008; Buenafuentes de la Mata 2010; Orduña 2019; Barrios Rodríguez 2022) está de 
acuerdo en que la designación ${ }^{5}$ es una característica principal del compuesto sintagmático. Sin embargo, la designación es un concepto abstracto y, hasta el momento, salvo en Barrios Rodríguez (2022), no se han establecido pruebas definitivas que distingan claramente un compuesto sintagmático de una locución nominal.

García-Page (2008) señala que los compuestos sintagmáticos, a diferencia de las locuciones, tienen unos esquemas sintácticos rígidos. Sin embargo, no observamos relevancia en las otras características que ofrece sobre los compuestos sintagmáticos, pues tras afirmar sobre ellos que tienen significado transparente, asegura inmediatamente después que «son numerosos los compuestos idiomáticos o semiidiomáticos» (García-Page 2008: 111).

También señala que la simplificación gráfica es característica de los compuestos sintagmáticos, sin embargo, inmediatamente después afirma: «Este proceso de coalescencia o de síntesis de formas analíticas también se efectúa en las locuciones» (García-Page 2008: 114). Estas breves menciones a uno de los trabajos más exhaustivos sobre el tema ilustran lo arduo de la clasificación y delimitación de los distintos tipos de construcciones nominales. Esta tarea, ya muy compleja de por sí, no se ve favorecida por la ingente cantidad de publicaciones, muchas de las cuales presentan eternos listados de características que admiten buena parte de las construcciones, con la particularidad de que dichas características no resultan útiles para la resolución de los problemas teóricos porque no son discriminatorias.

\subsection{Definición ostensiva: distinción entre locuciones nominales y compuestos sintag- máticos}

Barrios Rodríguez (2022) señala que los compuestos sintagmáticos designan entidades, individuos u objetos, y también hace referencia a la opcionalidad del sentido figurado en este tipo de construcciones. Sobre las locuciones nominales afirma que tienden a asociarse a un sentido figurado complejo que señala propiedades que frecuentemente son difíciles de definir y que pueden ser clasificadas como designativas (patata caliente, 'problema de difícil solución'), predicativas (cardo borriquero, 'muy feo'), infinitivas (coser y cantar, 'facilidad') y pluricategoriales (la niña de sus ojos, 'la persona preferida').

La propuesta de Barrios Rodríguez (2022) no niega que las locuciones nominales también puedan ser designativas (patata caliente designa un problema, es decir, funciona como un sustantivo). No obstante, señala que esa designación se realiza mediante un proceso de abstracción. Por tanto, propone la definición ostensiva ${ }^{6}$ como criterio principal para diferenciar la locución nominal del compuesto sintagmático.

Teniendo en cuenta lo propuesto por Barrios Rodríguez, perrito caliente, tocino de cielo, ojo de buey, etc., se clasificarían como compuestos sintagmáticos porque permiten la definición ostensiva: eso es un tocino de cielo. Construcciones como patata caliente, 'problema'; alto cargo, 'persona poderosa'; cuatro ojos, 'persona con gafas de vista'; tía buena, 'mujer atractiva', designan entidades de la realidad, personas u objetos. Sin embargo, no admiten la definición ostensiva: ?eso es un alto cargo, ?eso es un cuatro ojos, ?eso es una tía buena. Si bien estas construcciones son correctas sintácticamente, no expresan ni son capaces de mostrar mediante la ostensión el significado de las construcciones. Es decir, señalar a una

5 La prueba semántica de la designación consiste en hacer referencia a personas, cosas y otras entidades del mundo real que son reconocibles.

6 La definición ostensiva consiste en referirse a algo del mundo real señalando con el dedo (eso es un caracol). 
persona con gafas y decir eso es un cuatro ojos no significa que se esté haciendo uso de una expresión referencial, pues esa misma persona puede ser un alto cargo, un cuatro ojos y un cardo borriquero (todo a la vez). Como vemos, esta propuesta desecha la valoración de la presencia o ausencia de idiomaticidad en la construcción, ya que es un criterio que no causa conflictos en la definición ostensiva.

La prueba de la definición ostensiva es el colofón para distinguir de manera satisfactoria las locuciones nominales de los compuestos sintagmáticos, ya que, como hemos visto, las locuciones no admiten este tipo de definición. Según apunta Barrios Rodríguez (2022), tanto ella misma como García-Page y Buenafuentes de la Mata ya venían señalando que las construcciones nominales que funcionan como un sustantivo y sirven para designar, deberían clasificarse como compuestos sintagmáticos.

Conviene destacar que algunos ejemplos clasificados por Orduña (2019: 629) como compuestos sintagmáticos, bajo la prueba de la definición ostensiva no podrían clasificarse como tales: hijo de papi, mamitis aguda, negocios raros, buenas maneras.

\subsection{Tipologías de las construcciones nominales: designación vs. predicación}

Las locuciones nominales se han clasificado tradicionalmente según Casares (1969) como denominativas, singulares e infinitivas. Las locuciones denominativas son las que sirven para nombrar una cosa, persona o animal (tren correo). Sobre las locuciones singulares afirma que no se diferencian particularmente de las denominativas, pero ofrecen la particularidad de parecerse más al nombre propio que al nombre común (la carabina de Ambrosio, la purga de Benito). Las locuciones infinitivas se distinguen de las otras nominales porque lo sustantivo en ellas son los nombres infinitivos (coser y cantar, pedir peras al olmo).

La clasificación de Casares constituye la primera y más sistemática de las locuciones nominales, aunque cabe recoger el apunte de García-Page sobre una subdivisión dentro de las nominales infinitivas:

Suponemos que las infinitivas de Casares pueden ser, fundamentalmente, de tres tipos:
nominales, si su funcionamiento es puramente nominal [...]; verbales, si equivalen fun-
cionalmente a un verbo [...]; adjetivales, si hacen el oficio de un adjetivo [...]. Casares no
advirtió la existencia de locuciones con infinitivo inflexible como al correr de la pluma
y iHay que ver!, que habría que analizar como adverbial y exclamativa, respectivamente
(García-Page 2008: 91).

Una vez expuestos los distintos tipos de locuciones nominales, resulta, a nuestro juicio, enormemente sorpresivo, y está fuera de toda previsión, no hallar en ninguna de las distintas y no pocas clasificaciones de la bibliografía la distinción entre las locuciones nominales que pueden tener una función designativa y una función predicativa. Únicamente Casares (1969) hace cierta referencia a ello de manera completamente indirecta, y no es hasta una publicación de Barrios Rodríguez (2022) donde se establece una clara distinción entre la designación y la predicación en las locuciones nominales.

Tampoco encontramos referencia a las locuciones nominales predicativas en GarcíaPage. Sin embargo, este autor sí ha estudiado las construcciones que tienen un primer componente designativo y un segundo componente predicativo (noticia bomba) (GarcíaPage 2011). El estudio de este autor es especialmente relevante, ya que concluye afirmando 
que las construcciones con un componente designativo y otro predicativo son sintagmas en aposición, es decir, productos de la sintaxis y no de la fraseología. En la presente investigación defendemos que este tipo de construcciones son una categoría especial dentro de las locuciones nominales. Esta cuestión se tratará con mayor detalle en $§ 4.4$.

\section{CORPUS: CLASIFICACIÓN Y ANÁLISIS DE CONSTRUCCIONES NOMINA- LES}

\subsection{Clasificación de compuestos sintagmáticos}

Aplicada la prueba de la definición ostensiva con resultado positivo en los ejemplos 1-20 del corpus (véase Tabla 1), apreciamos que dicha prueba es efectiva para concretar el funcionamiento como expresión referencial de los compuestos sintagmáticos. Por otra parte, la presencia o ausencia de idiomaticidad no ha sido problemática ni relevante en el análisis de los ejemplos, por tanto, nos inclinamos a concluir que la idiomaticidad es una característica que no debería ser usada como objeto de análisis para clasificar los distintos tipos de construcciones nominales.

Hemos documentado una gran cantidad de compuestos sintagmáticos totalmente idiomáticos, es por ello que no estamos de acuerdo con una aseveración general de distintos autores, entre ellos, la RAE y la ASALE (2009: 743), que afirman que «los compuestos sintagmáticos suelen ser transparentes (ciudad dormitorio, decreto ley, relación madre-hija), si bien algunos lo son parcialmente (hombre rana, tren bala)». Además, aceptado lo que defendemos en $\S 3.2$, creemos que la clasificación de compuestos sintagmáticos transparentes de la RAE y la ASALE no es adecuada, ya que ciudad dormitorio y relación madre-hija no admiten la definición ostensiva por tratarse de sustantivos absolutamente abstractos y que no permiten su uso como expresiones referenciales.

La clasificación de los ejemplos 1-9 y 11-19 del corpus como compuestos sintagmáticos no genera dudas, en el sentido de que todos esos ejemplos se comportan de la misma manera: designan objetos, cosas o entidades de la vida real, permiten la definición ostensiva y pueden funcionar como expresiones referenciales. No obstante, reconocemos que existen otros compuestos que pueden generar más dudas; en el caso de campo santo (ej. 10) observamos que la definición ostensiva puede resultar más compleja por el hecho de tratarse de un lugar de cierta extensión y cuyo principio y final puede no ser del todo evidente. A pesar de esto, consideramos que en estos casos la definición ostensiva sí funciona en este tipo de construcciones, pues tienen unos elementos suficientemente característicos como para admitirla (eso es un campo santo) y presentan un comportamiento idéntico al de los demás compuestos sintagmáticos.

También hemos hallado un ejemplo de compuesto sintagmático complejo: patas de gallo (ej. 20), 'arrugas junto a los ojos'. Esta construcción se refiere a un estado de la piel que unas personas experimentan y otras no, es por ello que es dudosa la efectividad de la definición ostensiva en esta construcción. Sin embargo, tras cotejar el funcionamiento de esta construcción con el del sustantivo arruga, que sí permite la definición ostensiva, nos inclinamos a clasificar patas de gallo como compuesto sintagmático por funcionar como una entidad que permite el uso como expresión referencial y por no tratarse de un sustantivo abstracto. Por otra parte, cuando la gente se ríe siempre aparecen patas de gallo, en este caso es un estado momentáneo de la piel y puede parecer similar a la locución piel de gallina por admitir un uso dudoso de 
dicha construcción como expresión referencial. Por todo esto, reconocemos la complejidad de este ejemplo por estar a caballo entre un compuesto sintagmático y una locución designativa. No obstante, este tipo de compuestos sintagmáticos complejos son escasos.

Algunas construcciones nominales son polisémicas, es por ello por lo que no debería ser problemático clasificar la misma construcción más de una vez. Por ejemplo, pata de gallo puede significar 'arruga' o 'planta'. En este caso se clasificaría pata de gallo dos veces como compuesto sintagmático polisémico con sus correspondientes significados claramente diferenciados. Hay otros ejemplos polisémicos, como reina mora, que pueden clasificarse incluso en categorías distintas. En las construcciones las plumas de la reina mora son hermosísimas y buenos días, reina mora, encontramos un compuesto sintagmático (ave) y una locución nominal (apelativo) respectivamente. El análisis de los ejemplos propuestos ilustran que una misma construcción puede ser polisémica y al mismo tiempo pluricategorial. El hecho de que una construcción nominal pueda ser pluricategorial también es indicativo de la escasa fiabilidad de los esquemas sintácticos que proponen algunos autores para clasificar y distinguir las distintas construcciones nominales.

Tabla 1: Ejemplos de compuestos sintagmáticos

\begin{tabular}{|l|l|l|}
\hline & Compuesto sintagmático & \multicolumn{1}{c|}{ Significado } \\
\hline 1 & pez mujer & manatí, mamífero marino \\
\hline 2 & vaca marina & manatí, mamífero marino \\
\hline 3 & oro negro & petróleo \\
\hline 4 & reina mora & ave de plumas azules \\
\hline 5 & vara larga & pica para guiar toros \\
\hline 6 & tocino de cielo & postre de almíbar y huevo \\
\hline 7 & salado negro & zagua, arbusto \\
\hline 8 & trompa marina & instrumento musical \\
\hline 9 & media águila & moneda mexicana \\
\hline 10 & campo santo & cementerio \\
\hline 11 & leche virginal & líquido cosmético \\
\hline 12 & ropa vieja & guisado y restos de la olla \\
\hline 13 & palo cortado & vino de Jerez \\
\hline 14 & silla volante & carruaje de dos ruedas \\
\hline 15 & animal de bellota & cerdo \\
\hline 16 & lengua de gato & chocolatina alargada \\
\hline 17 & lengua de vaca & planta africana \\
\hline 18 & papel de aluminio & fina lámina de aluminio \\
\hline 19 & espejo de los incas & obsidiana \\
\hline 20 & patas de gallo & arrugas de los ojos \\
\hline
\end{tabular}

\subsection{Clasificación de locuciones nominales designativas}

En la presente clasificación de locuciones nominales designativas podemos observar que todas ellas funcionan siempre como sustantivos, de la misma forma que lo hacen los compuestos sintagmáticos. Sin embargo, estas locuciones nunca pueden usarse como expresiones referenciales y tampoco permiten la definición ostensiva, mientras que como hemos analizado anteriormente, los compuestos sintagmáticos sí que lo permiten. 
A pesar de los numerosos y muy diversos derroteros que ha tomado la bibliografía para tratar de distinguir, sin demasiado éxito, los compuestos sintagmáticos de las locuciones nominales designativas, la prueba de la definición ostensiva ha resultado muy útil para la clasificación y distinción de construcciones: hemos clasificado palo cortado (ej. 13) como compuesto sintagmático porque permite la definición ostensiva siendo su significado 'vino de Jerez'. Por otra parte, mano de santo (ej. 34, véase Tabla 2), construcción que hemos clasificado como locución nominal designativa, no permite la definición ostensiva ni su uso como expresión referencial porque muchas cosas distintas pueden ser mano de santo. Similar es lo que ocurre con mal de amores (ej. 39), es una locución nominal designativa que significa 'desamor', es decir, hace referencia a un sustantivo totalmente abstracto.

Los tres ejemplos analizados en el anterior párrafo se pueden emplear en una única oración (véase Ilustración 1) para esgrimir la diferencia entre compuestos sintagmáticos y locuciones nominales designativas que defendemos.

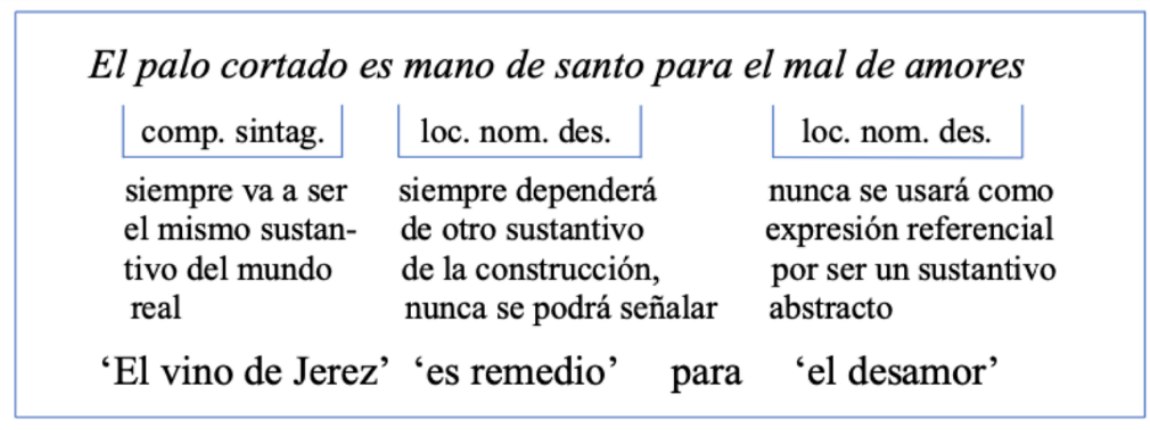

Ilustración 1: Oración con un compuesto sintagmático y dos locuciones

Se podría decir que el remedio para el mal de amores es el vino de Jerez: el palo cortado es mano de santo para el mal de amores. El palo cortado siempre va a ser vino de Jerez. Sin embargo, hay multitud de cosas que pueden ser mano de santo, por lo que mano de santo funciona como un sustantivo completamente abstracto y dependiente de otro, funcionamiento totalmente opuesto al de los compuestos sintagmáticos. Por su parte, mal de amores también funcionará siempre como un sustantivo abstracto y tendrá las mismas restricciones que mano de santo.

Tabla 2: Ejemplos de locuciones nominales designativas

\begin{tabular}{|l|l|l|l|l|}
\hline \multicolumn{1}{|c|}{$\begin{array}{c}\text { Locución } \\
\text { nominal }\end{array}$} & \multicolumn{1}{|c|}{ Sustantivo } & \multicolumn{1}{|c|}{ Paráfrasis } & \multicolumn{1}{c|}{ Ejemplo de uso } \\
\hline 21 & quinta columna & agrupación & $\begin{array}{l}\text { grupo de personas que ac- } \\
\text { túan secretamente con el } \\
\text { enemigo }\end{array}$ & $\begin{array}{l}\text { Los deleznables traidores de } \\
\text { la quinta columna }\end{array}$ \\
\hline 22 & mesa redonda & reunión & $\begin{array}{l}\text { reunión de personas con } \\
\text { conocimientos sobre un } \\
\text { tema }\end{array}$ & $\begin{array}{l}\text { La mesa redonda se celebró } \\
\text { ayer }\end{array}$ \\
\hline
\end{tabular}




\begin{tabular}{|c|c|c|c|c|}
\hline & $\begin{array}{c}\text { Locución } \\
\text { nominal }\end{array}$ & Sustantivo & Paráfrasis & Ejemplo de uso \\
\hline 23 & trato doble & fraude & $\begin{array}{l}\text { engaño para obtener bene- } \\
\text { ficio de otra persona }\end{array}$ & $\begin{array}{l}\text { Aquel trato doble rompió su } \\
\text { amistad }\end{array}$ \\
\hline 24 & puertas giratorias $^{7}$ & corrupción & $\begin{array}{l}\text { abuso de poder que consis- } \\
\text { te en ofrecer los mejores } \\
\text { puestos de trabajo en em- } \\
\text { presas privadas a personas } \\
\text { que dejan la política }\end{array}$ & $\begin{array}{l}\text { En España estamos hartos } \\
\text { de las puertas giratorias }\end{array}$ \\
\hline 25 & oferta del día & promoción & $\begin{array}{l}\text { promoción especial diaria } \\
\text { en un comercio }\end{array}$ & Compré la oferta del día \\
\hline 26 & baño maría & procedimiento & $\begin{array}{l}\text { procedimiento culinario } \\
\text { para calentar el contenido } \\
\text { de un recipiente }\end{array}$ & Cocinarlo al baño maría \\
\hline 27 & sueño dorado & deseo, aspiración & $\begin{array}{l}\text { deseo o aspiración de lo- } \\
\text { grar algún objetivo }\end{array}$ & $\begin{array}{l}\text { El sueño dorado de todo te- } \\
\text { nista es ganar Wimbledon }\end{array}$ \\
\hline 28 & $\begin{array}{l}\text { piedra en el ca- } \\
\text { mino }\end{array}$ & $\begin{array}{l}\text { problema, esco- } \\
\text { llo }\end{array}$ & problema, escollo & $\begin{array}{l}\text { Las colocaciones son una } \\
\text { piedra en el camino para al- } \\
\text { gunos gramáticos }\end{array}$ \\
\hline 29 & luz verde & $\begin{array}{l}\text { consentimiento, } \\
\text { permiso }\end{array}$ & permiso para realizar algo & $\begin{array}{l}\text { Nos dieron luz verde para } \\
\text { comenzar la obra }\end{array}$ \\
\hline 30 & vacas flacas & escasez & escasez & Son tiempos de vacas flacas \\
\hline 31 & línea caliente ${ }^{8}$ & servicio & servicio telefónico sexual & $\begin{array}{l}\text { Miguel es un asiduo de la } \\
\text { línea caliente }\end{array}$ \\
\hline 32 & talón de Aquiles ${ }^{9}$ & $\begin{array}{l}\text { debilidad, vulne- } \\
\text { rabilidad }\end{array}$ & punto débil & $\begin{array}{l}\text { Carlos enfureció cuando } \\
\text { Andrea se fue, ella es su ta- } \\
\text { lón de Aquiles }\end{array}$ \\
\hline 33 & \begin{tabular}{|l|}
$\begin{array}{l}\text { verdades como } \\
\text { puños }\end{array}$ \\
\end{tabular} & realidades & realidades & $\begin{array}{l}\text { Paqui solo dijo verdades } \\
\text { como puños }\end{array}$ \\
\hline 34 & mano de santo & remedio & $\begin{array}{l}\text { remedio para revertir o } \\
\text { mejorar algo }\end{array}$ & $\begin{array}{l}\text { Aquel masaje fue mano de } \\
\text { santo para mi dolor de es- } \\
\text { palda }\end{array}$ \\
\hline 35 & patata caliente $^{10}$ & juego & $\begin{array}{l}\text { juego de varios jugadores } \\
\text { que se pasan un objeto }\end{array}$ & $\begin{array}{l}\text { Estuvimos toda la tarde ju- } \\
\text { gando a la patata caliente }\end{array}$ \\
\hline
\end{tabular}

7 La construcción puertas giratorias es una locución nominal designativa cuando se refiere a ‘corrupción', pero también puede ser un compuesto sintagmático que designa un tipo de mecanismo para que las personas accedan a determinados sitios.

8 Documentamos numerosos ejemplos de uso de línea caliente para referirse a 'servicio sexual telefónico', no obstante, cabe resaltar que es una construcción muy polisémica, ya que también hemos encontrado una gran cantidad de ejemplos que hacen referencia a un tipo de servicio telefónico directo, importante, inmediato, etc.

9 Recogemos talón de Aquiles con los sentidos de 'debilidad', 'vulnerabilidad', no obstante, documentamos múltiples ejemplos de uso de talón de Aquiles con el sentido de 'tendón de Aquiles'. Casi todos estos ejemplos se encuentran en prensa deportiva y suelen hacer referencia a una operación en esta parte del cuerpo. Por lo tanto, cuando se emplea esta construcción para referirse al tendón de Aquiles se tratará de un compuesto sintagmático. 10 Patata caliente es una construcción polisémica que puede significar 'juego' o 'problema'. En cualquier caso, ambos sentidos de la construcción constituyen una locución nominal designativa. 


\begin{tabular}{|c|c|c|c|c|}
\hline & $\begin{array}{c}\text { Locución } \\
\text { nominal }\end{array}$ & Sustantivo & Paráfrasis & Ejemplo de uso \\
\hline 36 & cortina de humo ${ }^{11}$ & distracción & $\begin{array}{l}\text { distracción para desviar la } \\
\text { atención sobre otro asunto }\end{array}$ & $\begin{array}{l}\text { Esta nueva polémica es una } \\
\text { cortina de humo para tapar } \\
\text { la corrupción }\end{array}$ \\
\hline 37 & pelos y señales $^{12}$ & detalles & $\begin{array}{l}\text { todos los detalles de algo } \\
\text { por insignificantes que } \\
\text { sean }\end{array}$ & $\begin{array}{l}\text { Habla mucho del rifirrafe, } \\
\text { pero no da pelos y señales }\end{array}$ \\
\hline 38 & sexto sentido & intuición & $\begin{array}{l}\text { intuición de alguien para } \\
\text { averiguar cosas que pasan } \\
\text { desapercibidas para los } \\
\text { demás }\end{array}$ & $\begin{array}{l}\text { Mi mujer tiene un sexto sen- } \\
\text { tido, siempre me descubre }\end{array}$ \\
\hline 39 & mal de amores & desamor & $\begin{array}{l}\text { sufrimiento que siente una } \\
\text { persona enamorada de otra } \\
\text { por ser un amor no corres- } \\
\text { pondido o ante una ruptura } \\
\text { amorosa }\end{array}$ & $\begin{array}{l}\text { El remedio para el mal de } \\
\text { amores es el chocolate }\end{array}$ \\
\hline 40 & cacao mental & confusión & $\begin{array}{l}\text { gran confusión que tiene } \\
\text { una persona sobre algo }\end{array}$ & $\begin{array}{l}\text { Cristina no distingue el de- } \\
\text { recho civil del penal, ¡me- } \\
\text { nudo cacao mental! }\end{array}$ \\
\hline
\end{tabular}

\subsection{Clasificación de locuciones nominales predicativas}

Las locuciones nominales predicativas son las que más importancia tienen en este estudio, por ello, siguiendo el método de clasificación de Barrios Rodríguez (2022) y su propuesta de funciones léxicas no estándar, hemos clasificado cuatro tipos de locuciones nominales predicativas mediante las cuatro funciones que menciona: la primera de ellas es LocPropPers (locución de propiedades de personas), la segunda es LocPropPersPers (locución de propiedades de personas en relación con otras personas), la tercera es LocPropPerEntSit (locución de propiedades de personas, entidades y situaciones) y la cuarta es LocPropEntSit (locución de propiedades de entidades y situaciones).

Las locuciones nominales predicativas que describen propiedades de personas, en total oposición a las locuciones nominales designativas, funcionan exclusivamente como adjetivos. Como se puede comprobar en los ejemplos de uso de la Tabla 3, todas las locuciones clasificadas permitirían la sustitución por su correspondiente adjetivo o paráfrasis semántica: ¿cómo te llamas, buena alhaja?, ¿cómo te llamas, pícara?

11 La construcción cortina de humo, que clasificamos como locución nominal designativa cuando se refiere a 'distracción', se distingue claramente de esa misma expresión cuando se refiere a 'masa densa de humo provocada para ocultarse del enemigo'.

12 Si bien es cierto que pelos y señales se usa mayoritariamente antecedida por la preposición con, lo que la convertiría en una locución adverbial, hemos documentado algunos ejemplos que emplean la construcción con el verbo dar: me dio pelos y señales. Por lo tanto, la consideramos locución nominal. 
Tabla 3: Ejemplos de LocPropPers

\begin{tabular}{|l|l|l|l|l|}
\hline & Locución nominal & \multicolumn{1}{|c|}{ Adjetivo } & \multicolumn{1}{|c|}{ Paráfrasis } & \multicolumn{1}{|c|}{ Ejemplo de uso } \\
\hline 41 & alma de cántaro & ingenuo & persona muy ingenua & $\begin{array}{l}\text { iCómo puedes decir eso, } \\
\text { alma de cántaro! }\end{array}$ \\
\hline 42 & buena alhaja & pícaro & $\begin{array}{l}\text { persona astuta, viciosa y tra- } \\
\text { viesa }\end{array}$ & $\begin{array}{l}\text { iCómo te llamas, buena } \\
\text { alhaja? }\end{array}$ \\
\hline 43 & ratón de biblioteca & empollón & $\begin{array}{l}\text { tudiosa } \\
\text { África está siempre es- } \\
\text { tudiando, parece un ra- } \\
\text { tón de biblioteca }\end{array}$ \\
\hline 44 & perro viejo & $\begin{array}{l}\text { experimentado, } \\
\text { experto }\end{array}$ & $\begin{array}{l}\text { persona que ha aprendido } \\
\text { mucho con el paso del tiempo }\end{array}$ & $\begin{array}{l}\text { Juanmari es perro viejo } \\
\text { en esto de la Bibliogra- } \\
\text { fía }\end{array}$ \\
\hline 46 & alma en pena & afectado & $\begin{array}{l}\text { persona afectada emocional- } \\
\text { mente a causa de algún suce- } \\
\text { so o noticia }\end{array}$ & $\begin{array}{l}\text { La noticia de su muerte } \\
\text { me dejó muy mal cuerpo }\end{array}$ \\
\hline 47 & pobre y soberbio & pretencioso & $\begin{array}{l}\text { persona pobre que oculta y } \\
\text { niega su situación }\end{array}$ & $\begin{array}{l}\text { Raúl es pobre y soberbio } \\
\text { desde lo de su mujer }\end{array}$ \\
\hline 48 & animal de bellota & tonto & $\begin{array}{l}\text { persona de poco entendimien- } \\
\text { to }\end{array}$ & $\begin{array}{l}\text { iQué animal de bellota } \\
\text { estás hecho! }\end{array}$ \\
\hline 49 & pájaro de cuenta & $\begin{array}{l}\text { pícaro, aprove- } \\
\text { chado, } \\
\text { sinvergüenza }\end{array}$ & $\begin{array}{l}\text { persona a la que hay que tra- } \\
\text { tar con cuidado por su forma } \\
\text { de actuar }\end{array}$ & $\begin{array}{l}\text { Ignóralos, son pájaros } \\
\text { de cuenta con asuntos } \\
\text { con la justicia }\end{array}$ \\
\hline 50 & bala perdida & juerguista & $\begin{array}{l}\text { persona muy asidua de la fies- } \\
\text { ta y diversión }\end{array}$ & Alba es una bala perdida \\
\hline
\end{tabular}

Las locuciones nominales predicativas que describen propiedades de personas en relación con otras personas funcionan del mismo modo que las que describen propiedades de personas, pero se distinguen de ellas porque no pueden hacer referencia a una característica independiente de una persona, siempre describirán propiedades dependientes de otra u otras personas: farolillo rojo (ej. 54, véase Tabla 4), 'persona o equipo que ocupa la última posición en una competición'. Es decir, una persona no puede ser un farolillo rojo per se, sino que necesita que otras personas participen en la competición para poder serlo.

Tabla 4: Ejemplos de LocPropPersPers

\begin{tabular}{|l|l|l|l|l|}
\hline & Locución nominal & \multicolumn{1}{|c|}{ Adjetivo } & \multicolumn{1}{|c|}{ Paráfrasis } & \multicolumn{1}{c|}{ Ejemplo de uso } \\
\hline 51 & abogado del diablo & defensor & $\begin{array}{l}\text { persona que defiende y con- } \\
\text { tradice una idea o proposi- } \\
\text { ción que ha manifestado otra } \\
\text { persona }\end{array}$ & $\begin{array}{l}\text { Pedro hizo de abogado } \\
\text { del diablo al apoyar la } \\
\text { pena de muerte }\end{array}$ \\
\hline 52 & cabeza de turco & $\begin{array}{l}\text { inculpado, cul- } \\
\text { pable }\end{array}$ & $\begin{array}{l}\text { persona que paga las culpas } \\
\text { de otras personas }\end{array}$ & $\begin{array}{l}\text { Esa pobre mujer solo es } \\
\text { la cabeza de turco de la } \\
\text { trama }\end{array}$ \\
\hline 53 & palomo cojo & homosexual & $\begin{array}{l}\text { hombre que se siente atraído } \\
\text { por otros hombres }\end{array}$ & $\begin{array}{l}\text { Manuel es un palomo } \\
\text { cojo }\end{array}$ \\
\hline
\end{tabular}




\begin{tabular}{|l|l|l|l|l|}
\hline & Locución nominal & \multicolumn{1}{|c|}{ Adjetivo } & \multicolumn{1}{|c|}{ Paráfrasis } & \multicolumn{1}{|c|}{ Ejemplo de uso } \\
\hline 54 & farolillo rojo & último, peor & $\begin{array}{l}\text { persona o equipo que ocupa } \\
\text { la última posición en una } \\
\text { competición }\end{array}$ & $\begin{array}{l}\text { Roque es farolillo } \\
\text { rojo de esta competición }\end{array}$ \\
\hline 55 & oveja negra & peor & el peor de un grupo o familia & $\begin{array}{l}\text { Patricia es la oveja ne- } \\
\text { gra de la familia }\end{array}$ \\
\hline 56 & oveja descarriada & inmoral & $\begin{array}{l}\text { persona de un grupo o fami- } \\
\text { lia que contrasta con el resto } \\
\text { por su mala conducta }\end{array}$ & $\begin{array}{l}\text { Jorge es la oveja desca- } \\
\text { rriada de mi grupo de } \\
\text { amigos }\end{array}$ \\
\hline 57 & ciego de amor & $\begin{array}{l}\text { enamorado, } \\
\text { apasionado }\end{array}$ & $\begin{array}{l}\text { persona irremediablemente } \\
\text { enamorada de otra }\end{array}$ & $\begin{array}{l}\text { Manuel está ciego de } \\
\text { amor }\end{array}$ \\
\hline 58 & buen samaritano & $\begin{array}{l}\text { solidario, } \\
\text { humanitario }\end{array}$ & $\begin{array}{l}\text { persona que ayuda a otras } \\
\text { desinteresadamente }\end{array}$ & $\begin{array}{l}\text { Carmelo es muy buen } \\
\text { samaritano }\end{array}$ \\
\hline
\end{tabular}

Las locuciones nominales predicativas que describen propiedades de personas, entidades y situaciones son las menos numerosas de las locuciones nominales predicativas. Este tipo de locuciones tienen la particularidad de que designan propiedades tanto de personas como de cosas: pañuelo de lágrimas (ej. 59, véase Tabla 5) puede ser una persona que consuela a otra o un objeto que realiza la misma función: Ariel es mi pañuelo de lágrimas, tus brazos son mi pañuelo de lágrimas.

Tabla 5: Ejemplos de LocPropPersEntSit

\begin{tabular}{|l|l|l|l|l|}
\hline & Locución nominal & Adjetivo & Paráfrasis & Ejemplo de uso \\
\hline 59 & $\begin{array}{l}\text { pañuelo/paño de lá- }_{\text {grimas }^{13}} \\
\text { consolador }\end{array}$ & $\begin{array}{l}\text { (persona o cosa) que consuela a } \\
\text { una persona }\end{array}$ & $\begin{array}{l}\text { Ariel es mi pañuelo de } \\
\text { lágrimas; este pañuelo es } \\
\text { mi paño de lágrimas }\end{array}$ \\
\hline 60 & poca cosa & insuficiente & $\begin{array}{l}\text { (persona o cosa) que no cumple } \\
\text { unas determinadas expectativas }\end{array}$ & $\begin{array}{l}\text { Marta es muy poca cosa } \\
\text { para mí; este puesto de } \\
\text { trabajo es poca cosa para } \\
\text { mí }\end{array}$ \\
\hline
\end{tabular}

Las locuciones nominales predicativas que describen propiedades de entidades y situaciones funcionan del mismo modo que todas las predicativas, con la salvedad de que no son aplicables a personas: la casa de tócame Roque (ej. 62, véase Tabla 6) no hace referencia en todos los casos a una casa, sino a un lugar, es por ello que es una locución nominal predicativa que designa propiedades de lugares.

Tabla 6: Ejemplos de LocPropEntSit

\begin{tabular}{|l|l|l|l|l|}
\hline & Locución nominal & Adjetivo & Paráfrasis & Ejemplo de uso \\
\hline 61 & olla de grillos & caótico & $\begin{array}{l}\text { lugar con gran desorden y al- } \\
\text { boroto }\end{array}$ & $\begin{array}{l}\text { Este país es una olla de } \\
\text { grillos }\end{array}$ \\
\hline
\end{tabular}

13 Documentamos ambas variantes.

14 Tomado de un ejemplo de uso real: «este pañuelo blanco que ella me arrojó al partir, es mi paño de lágrimas» (https://xurl.es/a322m, 12/11/2021). 


\begin{tabular}{|l|l|l|l|l|}
\hline 62 & Locución nominal & Adjetivo & Paráfrasis & Ejemplo de uso \\
Roque de tócame & caótico & $\begin{array}{l}\text { lugar con gran desorden y mu- } \\
\text { cha gente }\end{array}$ & $\begin{array}{l}\text { La clase parece la casa de } \\
\text { tócame Roque }\end{array}$ \\
\hline 63 & el séptimo cielo & placentero & $\begin{array}{l}\text { lugar extremadamente placen- } \\
\text { tero }\end{array}$ & $\begin{array}{l}\text { Este hotel es el séptimo } \\
\text { cielo }\end{array}$ \\
\hline 64 & subida de tono & inapropiado & $\begin{array}{l}\text { cosa inapropiada, grosera u } \\
\text { obscena }\end{array}$ & $\begin{array}{l}\text { Declaraciones muy subi- } \\
\text { das de tono }\end{array}$ \\
\hline
\end{tabular}

\subsection{Clasificación de locuciones nominales complejas}

En el proceso de clasificación de locuciones nominales designativas y predicativas hemos hallado cierto número de construcciones complejas que dificultan la clasificación. En primer lugar, hemos observado que hay una serie de construcciones cuyo primer componente es transparente y funciona como un sustantivo, y el segundo componente es predicativo. Algunos ejemplos de esto son negocio redondo (ej. 71, véase Tabla 7), estudiante modelo (ej. 65), diálogo de besugos (ej. 66) y premio gordo (ej. 69). En todos estos casos el primer sustantivo funciona de forma transparente (aunque se da una distinta gradación en la transparencia de determinadas construcciones), sin embargo, el segundo componente tiene una función fuertemente predicativa que le otorga una parte esencial del significado a las construcciones. Si nos detenemos en el ejemplo diálogo de besugos, podemos comentar que siempre se va a tratar de un diálogo o una conversación. Sin embargo, no creemos procedente clasificarla como una locución nominal designativa porque se diferencia claramente de algunos ejemplos clasificados en la Tabla 2 como pelos y señales, 'detalles' (ej. 37); cacao mental, 'confusión' (ej. 40), que funcionan como sustantivos abstractos sin predicados. Diálogo de besugos no solamente hace referencia a un diálogo o una conversación, sino que las propiedades torpe o absurda que contiene la construcción son totalmente necesarias para la correcta interpretación de la locución. Es por ello por lo que clasificamos aparte este tipo de locuciones nominales que tienen un indudable componente predicativo, a pesar de que designan un sustantivo abstracto.

En segundo lugar, hemos identificado otro tipo de construcciones que se comportan de manera muy particular: noticia bomba (ej. 72), aunque parece de idéntica estructura y funcionamiento que las descritas en el anterior párrafo, es muy distinta. Por una parte, noticia bomba presenta la problemática planteada en el anterior párrafo cuando se emplea en construcciones como ;menuda noticia bomba! En este caso, noticia está funcionando como un sustantivo transparente. Sin embargo, hemos documentado que esta construcción tiene otros usos como en su declaración no es noticia bomba, o en su ruptura es noticia bomba. En estos casos noticia bomba funciona exclusivamente como un adjetivo, pues se puede sustituir por sorprendente: su ruptura es sorprendente.

Sobre estas locuciones nominales complejas cabe destacar que Barrios Rodríguez (2022) ya advierte el doble comportamiento en algunas construcciones. La autora clasifica la locución lágrimas de cocodrilo como locución nominal pluricategorial, y se refiere a este último tipo de locuciones como construcciones que designan y predican simultáneamente.

Consideramos que la terminología que proponemos para estas construcciones que designan y predican de manera concomitante (locuciones nominales complejas) es más adecuada que la que propone Barrios Rodríguez (2022) (locuciones nominales pluricategoriales) por 
el siguiente motivo: el concepto pluricategoriales lo hemos reservado en esta investigación para esas locuciones que, dependiendo de la construcción en la que se encuentren, pueden ser locuciones nominales complejas (menuda noticia bomba) o locuciones nominales predicativas (su ruptura no es noticia bomba), como ya hemos señalado anteriormente, en este último ejemplo la locución no está predicando y designando simultáneamente, sino que únicamente predica.

Tabla 7: Ejemplos de locuciones nominales complejas

\begin{tabular}{|l|l|l|l|l|}
\hline & Locución nominal & Sustantivo & Adjetivo & Ejemplo de uso \\
\hline 65 & estudiante modelo & estudiante & ideal, perfecto & $\begin{array}{l}\text { Laura es la estudiante modelo de la } \\
\text { facultad }\end{array}$ \\
\hline 66 & $\begin{array}{l}\text { diálogo de besu- } \\
\text { gos }^{15}\end{array}$ & conversación & torpe & Aquello era un diálogo de besugos \\
\hline 67 & príncipe azul & hombre (pareja) & perfecto & Él es mi príncipe azul \\
\hline 68 & media naranja & pareja & perfecta & Está claro que es su media naranja \\
\hline 69 & premio gordo & premio & mejor, mayor & $\begin{array}{l}\text { Me ha tocado el premio gordo; eres } \\
\text { mi premio gordo }\end{array}$ \\
\hline 70 & ciudad fantasma & lugar & $\begin{array}{l}\text { desierto, poco ha- } \\
\text { bitado }\end{array}$ & $\begin{array}{l}\text { Aquella aldea parecía una ciudad } \\
\text { fantasma; Moscú es una ciudad fan- } \\
\text { tasma }\end{array}$ \\
\hline 71 & negocio redondo & negocio, trato & $\begin{array}{l}\text { beneficioso, lu- } \\
\text { crativo }\end{array}$ & $\begin{array}{l}\text { La compra de mascarillas fue un ne- } \\
\text { gocio redondo }\end{array}$ \\
\hline 72 & noticia bomba & $\begin{array}{l}\text { noticia, declara- } \\
\text { ción }\end{array}$ & $\begin{array}{l}\text { sorprendente, in- } \\
\text { esperada }\end{array}$ & $\begin{array}{l}\text { Su afirmación fue noticia bomba; } \\
\text { hoy hemos publicado una noticia } \\
\text { bomba }\end{array}$ \\
\hline
\end{tabular}

\section{FUTURAS LÍNEAS DE INVESTIGACIÓN}

Las locuciones denominadas originalmente en este trabajo locuciones nominales complejas son las que presentan mayores problemas. Hemos ilustrado en el apartado anterior que este tipo de locuciones pueden presentar dos tipos de problemas distintos: el primer problema consiste en que el primer componente de la locución designe y el segundo predique, mientras que el segundo problema consiste en que una construcción funcione en algunos contextos como adjetivo y en otros como una locución con un primer componente designativo y un segundo componente predicativo.

Los resultados de nuestra clasificación de las distintas locuciones nominales permiten apreciar que estas locuciones nominales complejas son frecuentes y requieren una sistematización coherente que permita desarrollar una clasificación completa de los tipos de locuciones nominales. Es indiscutible que los ejemplos analizados en la Tabla 7 no pueden considerarse como locuciones nominales designativas o predicativas por lo versátiles que se muestran y por las diferencias que presentan respecto a las verdaderas locuciones nominales designativas y predicativas clasificadas en sus correspondientes tablas.

Las construcciones premio gordo (ej. 69, véase Tabla 7) y verdades como puños (ej. 33, véase Tabla 2) permiten ahondar en la dificultad que supone distinguir los distintos tipos

15 Documentamos la variante diálogo para besugos. 
de locuciones nominales. Premio gordo funciona como una de esas locuciones complejas que hemos analizado; su primer componente designa y el segundo predica. Por su parte, verdades como puños, a pesar de parecer una construcción idéntica (en la que verdades es transparente y como puños funcionaría como el adjetivo incontestables), tras el cotejo de numerosos ejemplos de uso de la construcción nos inclinamos a afirmar que verdades como puños no significa 'verdades incontestables', sino que es una auténtica locución nominal designativa que designa realidades. Este ejemplo muestra la complejidad de este tipo de locuciones y la necesidad de elaborar, como hemos comentado, una propuesta de formalización de estas construcciones.

\section{CONCLUSIONES}

A la vista de los resultados del análisis realizado sobre los compuestos sintagmáticos y las locuciones nominales designativas afirmamos que la prueba de la definición ostensiva ha tenido un carácter verdaderamente discriminatorio y nos ha permitido clasificar estos dos tipos de construcciones en todos los ejemplos propuestos. Además, la discusión bibliográfica desarrollada en las primeras páginas de esta investigación sobre los límites de las locuciones y las colocaciones, incidiendo en la característica de la fijación, ha permitido distinguir las locuciones nominales de las colocaciones nominales, y estas dos últimas de los compuestos sintagmáticos.

Teniendo en cuenta las construcciones analizadas y clasificadas en nuestro corpus, consideramos que es evidente la existencia de dos tipos de locuciones nominales: las designativas (que funcionan como sustantivos) y las predicativas (que funcionan como adjetivos). Sin embargo, a través del intento de demostrar la existencia de estos dos tipos de locuciones nominales claramente diferenciadas, hemos comprobado que es necesaria alguna categoría más para poder abarcar y clasificar todas las locuciones nominales. Concretamente hemos mostrado la existencia de las que hemos llamado locuciones nominales complejas, que tienen un primer componente que designa y un segundo componente que predica, características que impiden considerarlas locuciones designativas o predicativas por diferenciarse sustancialmente de ellas. Las locuciones nominales complejas son doblemente problemáticas porque además de funcionar de la manera descrita, en contextos específicos pueden funcionar exclusivamente como adjetivos y ser, por tanto, locuciones pluricategoriales.

\section{REFERENCIAS BIBLIOGRÁFICAS}

Barrios Rodríguez, M. A. (2015). Las colocaciones del español. Madrid: Arco/Libros.

Barrios Rodríguez, M. A. (2022). Las colocaciones nominales: una aproximación desde la Teoría Sentido-Texto. Santiago de Compostela: Verba. Anuario Galego de Filoloxía, Anexo 82.

Bosque, I. (2004). Redes. Diccionario combinatorio del español contemporáneo. Madrid: Ediciones SM.

Buenafuentes de la Mata, C. (2010). La composición sintagmática en español. San Millán de la Cogolla: Cilengua.

Bustos Gisbert, E. (1986). La composición nominal en español. Salamanca: Ediciones Universidad de Salamanca.

Casares, J. (1969). Introducción a la lexicografía moderna. Madrid: Consejo Superior de Investigaciones Científicas. 
Corpas Pastor, G. (1996). Manual de fraseología española. Madrid: Gredos.

García-Page, M. (2008). Introducción a la fraseología española. Estudio de las locuciones. Barcelona: Anthropos Editorial.

García-Page, M. (2011). «Hombre clave, hombre rana, ¿un mismo fenómeno?», Verba. Anuario Galego de Filoloxía, 38, pp. 127-170.

Koike, K. (2001). Colocaciones léxicas en el español actual: estudio formal y léxico-semántico. Universidad de Alcalá.

Mel'čuk, I. (1996). «Lexical functions: A tool for the description of lexical relations in a lexicon». Lexical functions in lexicography and natural language processing. Amsterdam: J. Benjamins, pp. 37-102.

Mel'čuk, I. (2001). Communicative organization in natural language: the semantic-communicative structure of sentences. Amsterdam: J. Benjamins.

Orduña López, J. L. (2019). «¿Locuciones nominales o compuestos sintagmáticos? A propósito del español del Valle de Aburrá». Íkala, Revista de Lenguaje y Cultura, 24, pp. 619-636.

Real Academia Española. (2001). Diccionario de la lengua española. Madrid: Espasa.

Real Academia Española y Asociación de Academias de la Lengua Española. (2009). Nueva gramática de la lengua española. Barcelona: Espasa.

Ruiz Gurillo, L. (2001). Las locuciones en español actual. Madrid: Arco/Libros.

Seco, M., O. Andrés y G. Ramos. (2004). Diccionario fraseológico documentado del español actual. Locuciones y modismos españoles. Madrid: Santillana. 